Original Research Paper

\title{
Irrigation System Using Hyperspectral Data and Machnie Learning Techniques for Smart Agriculture
}

\author{
Santhi Balachandran, Sourna Lakshmi and Nithya Rajendran \\ School of Computing, SASTRA Deemed University, Thanjavur, TamilNadu 613402, India
}

\author{
Article history \\ Received: 15-07-2019 \\ Revised: 12-09-2019 \\ Accepted: 13-03-2020 \\ Corresponding Author: \\ Santhi Balachandran \\ School of Computing, SASTRA \\ Deemed University, Thanjavur, \\ TamilNadu 613402, India \\ Email: shanthi@cse.sastra.edu
}

\begin{abstract}
Water is the main resource for agriculture. Management of water in agricultural field is a challenging process. To manage the water content in the agricultural field, smart irrigation system has been proposed by using fuzzy based decision support system on Hyperspectral Image benchmark dataset. Hyperspectral images are the process of collected and processed the images from electromagnetic spectrum. Recent studies show that hyperspectral images are very accurate in collecting the soil moistures value. Dataset is collected in five-day field of campaign the soil is the type of clayey slit and it is non vegetation. Hyperspectral datasets which consist of range value between 454 to $598 \mathrm{~nm}$. Value is gathered from the 285 hyperspectral snapshot camera recording images with 125 spectral bands with the spectral resolution of $4 \mathrm{~nm}$. Experimental results of this method achieve the accuracy of 0.98 . Hence the proposed method reduces the water wastage to an extent.
\end{abstract}

Keywords: Hyperspectral, Fuzzy Logic, Smart Farming

\section{Introduction}

In recent years, the efficient utilization of water in agriculture is the most important challenges in modern agriculture (Polak et al., 2017). In India, the rate of agriculture is poor, because of the nutrient contents and wastage of water or wrong irrigation practices. Smart agriculture system has been developed in now-a-days with the help of Hyperspectral images. This emerging technology is the first step for the application like agriculture, pharma and other fields (Zhong et al., 2015). Hyperspectral imaging has distinguished itself among the more developed systems used to gather extensive scale information about an object without physical or closer contact with the object and also used to identify the minerals and vegetation content in soil (Cao et al., 2017a). Hyperspectral sensors are used to collect remote images with the high resolution. It often captures hundreds of bands for each pixel. Pixel resolution is taken as $10 \mathrm{~nm}$ and it has dozens and hundreds of visible and infrared ranges (Riese and Keller, 2018). Each image is dividing into bands whereas all the bands do not contain the information. Only selective bands will contain the information. Irrelevant bands are available that decrease the classification accuracy (Khan et al., 2018). Soil moisture prediction model was mostly the study of different soil types such as clay, loamy, red soil, black soil etc. (Bandos et al., 2009). There are many other influencing factors like roughness, texture, mineral content is present in soil to analyze the soil moisture value but the result of soil moisture value is considered in the top layer of $5 \mathrm{~cm}$ (Zhong et al., 2006). Data from the soil sensor is used to create the decision model (Govender et al., 2007). The band selection is used to improve the performance of hyperspectral image classification (Breiman, 2017). Many methods to find the soil character and the soil types such as linear regression, Support Vector Machine (SVM), Decision tree. Support vector is mainly used for estimate the soil moisture content in hyperspectral images (Wark et al., 2007).

Fuzzy logic system has an ability to represent the subjective knowledge in mathematical model (Majone et al., 2013). The main objective of fuzzy based rules of inference is to find the amount of water required for the field that reduces the wastage of water content drastically. Numerous elements, for example, defective imaging optics, climatic disturbances, optical brightening impacts and sensor noise cause the corruption of the procure image quality, making spatial determination the costlier one and hardest to improve in imaging frameworks (Neamatollahi et al., 2017). Supervised Learning uses the hyperspectral data (Cao et al., 2017b). In fuzzy logic, the input and output relation are expressed by the set of linguistic rules. IF-THEN rules are employed to express conditional statements that constitute fuzzy logic (Martinez-Agirre et al., 2017). 


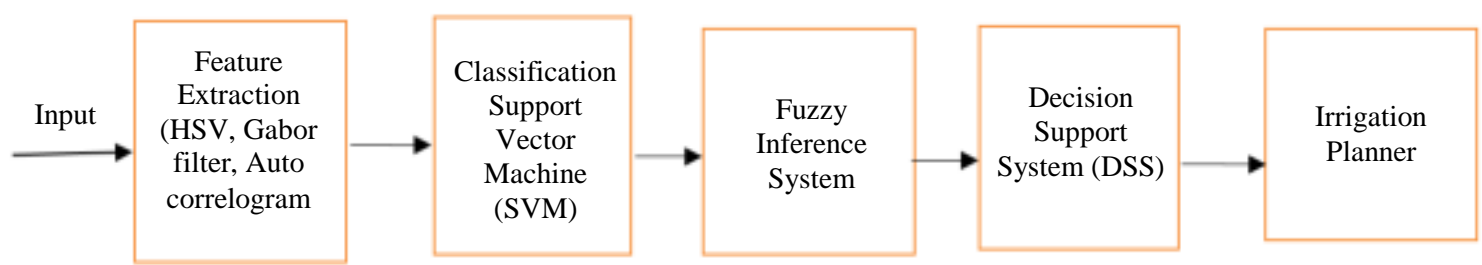

Fig. 1: Workflow diagram of proposed System

However fuzzy logic is used for prediction either yes/no or true/false based on the rules (Roseline et al., 2012). Choosing the good prediction is the complex process however assuming the simple linear regression may lead to poor prediction. Non-linear prediction is needed to improve the accuracy of soil moisture from hyperspectral data.

Mostly fuzzy based system is consisting of four parts such as fuzzifier, de-fuzzifier, inference system and rule generator (Webber et al., 2016). Hyperspectral imaging widely used in remote sensing applications, which involves complex surface measurements and detection of identical materials having fine spectral signatures (Palacios-Orueta and Ustin, 1998). This fuzzy system cannot be focus only on water management it also can be supported for selected crops. Based on the crop, rules are formed and the inputs are specially gathered for the selective crops (Jadhav and Patil, 2014). Input data are collected from the special dataset with the high-resolution images and with the high accurate data (Riese and Keller, 2018).

\section{Existing System}

The soil classification is the main area to increase the crop yield. Soil classification is carried out by Support Vector Machine that classifies the type of soil by extracting the features from the soil image. Fuzzy rules are collected based on the experience of farmers to design the rules for the irrigation management. The suggested fuzzy rules are used to reduce the water waste and make the system to react according to weather monitoring system. This system is a userfriendly since it releases the water according to the need of the land and crops.

\section{Proposed Work}

The Proposed methodology is the combination of wireless sensor network and fuzzy logic (i.e., Mamdani). Initially, noise has been removed from the input image using Gabor filter and low pass filter. Then, features are extracted from the image as statistical parameters like Auto correlogram, HSV Histogram, Standard deviation, Color moments, mean amplitude, energy, Temperature, Humidity and Wavelet transform. Finally, the irrigation quantity has been outputted based on the fuzzy rules. The overview of the proposed system is depicted in Fig. 1.

\section{Hyperspectral Datasets}

Hyperspectral Datasets is measured in five-day field over two weeks. Dataset is taken from snapshot of camera recording of 50 by 50 images with 125 spectral bands with the range from $450 \mathrm{~nm}$ to $950 \mathrm{~nm}$ with the resolution of $4 \mathrm{~nm}$. Soil sample is taken from the sensor with the radius of $15 \mathrm{~cm}$ and with the height of $20 \mathrm{~cm}$ (Riese and Keller, 2018).

\section{Classifications}

\section{Decision Tree}

Decision Tree is a statistical/machine learning technique for classification and regression. It is mainly used for interaction between the variables and new data. This method makes an accurate prediction for new data. Best accuracy value is calculated as 0.88861 in the fine tree method. Table 1 shows the decision tree types and their properties employed in this work.

\section{Support Vector Machine (SVM)}

Support Vector Machine is a selection method that compares the standard parameter set of discrete values, called the candidate set and takes the one that has the best classification accuracy. Best accuracy value is calculated as 0.55662 in the Fine Gaussian method compared with all the other SVM methods. Table 2 shows the SVM variants available in matlab toolbox.

\section{Linear Regression}

Learning a linear regression means estimating the values of coefficient that is used to represent the data. It is a machine learning model. Mainly it is used for finding the relationship between variables and forecasting. There are different regression models that it based on the relationship in data between dependent and in dependent variable.

\section{Robust Regression}

Regression analysis finds the relationship between one or more independent variable and the dependent variable. Robust regression method is designed for not affecting the violations underlying the data generating process.

\section{Stepwise Regression}

Stepwise regression is the combination of both forward and backward selection technique. It contains 
the multi variate selection procedure it will perform usually a better.

\section{Comparison of RMSE and $R^{2}$}

RMSE is an error value by performing the mean operation and whereas $\mathrm{R}^{2}$ means taking the square for all the mean values. Values are calculated using the regression learner app in MATLAB which includes all the regression models. RMSE and $\mathrm{R}^{2}$ valuehas been compared for 285 hyperspectral snapshots. This comparison gives the best and worst fit regression value from the band datasets. Classification is carried out with different feature selection in the ratio of 39,29 and $25,20,15,10$. Table 3 and 4 represents the feature selections.

Table 1: Decision tree types

\begin{tabular}{llll}
\hline Properties & Coarse tree & Medium tree & Fine tree \\
\hline Flexibility & Low & Medium & High \\
Splits & 4 & 20 & 100 \\
Prediction & Fast & Fast & Fast \\
\hline
\end{tabular}

Table 2: SVM variants

\begin{tabular}{|c|c|c|c|c|c|}
\hline \multirow[b]{2}{*}{ SVM Variants } & \multicolumn{2}{|c|}{ Prediction speed } & \multicolumn{2}{|c|}{ Memory usage } & \multirow[b]{2}{*}{ Interpretability } \\
\hline & Binary & Multiclass & Binary & Multiclass & \\
\hline Linear SVM & Fast & Medium & Medium & Medium & Easy \\
\hline Quadratic SVM & Fast & Slow & Medium & Large & Hard \\
\hline Cubic SVM & Fast & Slow & Medium & Large & Hard \\
\hline Fine Gaussian SVM & Fast & Slow & Medium & Large & Hard \\
\hline Medium Gaussian SVM & Fast & Slow & Medium & Large & Hard \\
\hline Coarse Gaussian SVM & Fast & Slow & Medium & Large & Hard \\
\hline
\end{tabular}

Table 3: Features selection 39, 29, 25

\begin{tabular}{|c|c|c|c|c|c|c|c|}
\hline \multicolumn{8}{|c|}{ Number of Features Selection } \\
\hline \multirow[b]{2}{*}{ Classification } & \multirow[b]{2}{*}{ METHOD } & \multicolumn{2}{|l|}{39} & \multicolumn{2}{|l|}{29} & \multicolumn{2}{|l|}{25} \\
\hline & & RMSE & $\mathrm{R}^{2}$ & RMSE & $\mathrm{R}^{2}$ & RMSE & $\mathrm{R}^{2}$ \\
\hline \multirow{3}{*}{ Decision Tree } & Fine Tree & 0.88861 & 0.94 & 1.0083 & 0.92 & 1.0094 & 0.92 \\
\hline & Medium Tree & 0.97815 & 0.93 & 1.0569 & 0.92 & 1.0383 & 0.92 \\
\hline & Coarse Tree & 1.3896 & 0.86 & 1.3155 & 0.87 & 1.3155 & 0.87 \\
\hline \multirow{4}{*}{ SVM } & Linear SVM & 1.5509 & 0.82 & 1.4992 & 0.83 & 1.5034 & 0.83 \\
\hline & Fine Gaussian & 0.5562 & 0.98 & 3.6366 & 0.01 & 1.6168 & 0.80 \\
\hline & Coarse Gaussian & 1.9092 & 0.73 & 1.5082 & 0.83 & 1.4431 & 0.84 \\
\hline & Medium Gaussian & 1.0225 & 0.92 & 1.4782 & 0.86 & 1.2803 & 0.88 \\
\hline \multirow{3}{*}{ Regression } & Linear & 1.4679 & 0.84 & 1.4354 & 0.85 & 1.4237 & 0.85 \\
\hline & Robust Linear & 1.742 & 0.77 & 1.711 & 0.78 & 1.704 & 0.78 \\
\hline & Stepwise Linear & 1.411 & 0.85 & 1.367 & 0.86 & 1.860 & 0.52 \\
\hline
\end{tabular}

Table 4: Features selection 20, 15, 10

\begin{tabular}{|c|c|c|c|c|c|c|c|}
\hline \multicolumn{8}{|c|}{ Number of features section } \\
\hline \multirow[b]{2}{*}{ Classification } & \multirow[b]{2}{*}{ METHOD } & \multicolumn{2}{|l|}{20} & \multicolumn{2}{|l|}{15} & \multicolumn{2}{|l|}{10} \\
\hline & & RMSE & $\mathrm{R}^{2}$ & RMSE & $\mathrm{R}^{2}$ & RMSE & $\mathrm{R}^{2}$ \\
\hline \multirow{4}{*}{ Decision Tree } & Fine Tree & 1.0093 & 0.92 & 0.9890 & 0.93 & 0.72258 & 0.96 \\
\hline & Medium Tree & 1.0383 & 0.92 & 1.0369 & 0.92 & 0.77034 & 0.96 \\
\hline & Coarse Tree & 1.3155 & 0.87 & 1.3155 & 0.87 & 1.2595 & 0.88 \\
\hline & Linear SVM & 3.5884 & 0.83 & 1.5209 & 0.83 & 1.7787 & 0.76 \\
\hline \multirow[t]{3}{*}{ SVM } & Fine Gaussian & 3.5884 & 0.03 & 3.3637 & 0.15 & 3.2494 & 0.21 \\
\hline & Coarse Gaussian & 1.4431 & 0.84 & 1.3608 & 0.87 & 1.6388 & 0.80 \\
\hline & Medium Gaussian & 1.2803 & 0.88 & 0.9890 & 0.93 & 0.72258 & 0.96 \\
\hline \multirow[t]{3}{*}{ Regression } & Linear & 1.4237 & 0.85 & 1.0369 & 0.92 & 0.77034 & 0.96 \\
\hline & Robust Linear & 1.704 & 0.78 & 1.3155 & 0.87 & 1.2595 & 0.88 \\
\hline & Stepwise Linear & 1.203 & 0.22 & 1.5209 & 0.83 & 1.7787 & 0.76 \\
\hline
\end{tabular}




\section{Comparison Result}

Table 5 shows the Comparison result of Classifications method shows less RMSE values and higher $\mathrm{R}^{2}$ values. Hence it is a suitable classifier for soil classification when compared with the previous methods. Experimental results show that SVM provides the better values compared with all the other classification mechanism.

\section{Fuzzy}

Fuzzy Logic is a logical system and it is an extended value of multi valued logic. It is the concept of set of fuzzy values and mainly used for decision making process. Input value is processed as manual partition and passed to the decision system. Then, Fuzzy is used for making the decision for the quantity of water that should serve for farming land. Based on the soil moisture value the quantity is decided. Fuzzy rules are formed based on the partition of data. At the time of execution, the rules are evaluated based on the quantity of water. Decision making system is range of Conditions as YES/NO or TRUE/FALSE or 0/1. Soil moisture is the important feature in the rules.

Table 6 shows the soil moisture range value. Based upon this soil moisture, the quantity of water has been decided. These data were measured in spectral band in various band measures. The box plot represents the upper and lower quartiles, while inside the line represents the median value. In Fig. 2, box plots shows how efficiently discriminate soil moisture such as low, medium and high using spectral band values.

The Fig. 3 shows the hyperspectral date for soil moisture. The plot represented by $\mathrm{x}$ and $\mathrm{y}$ values, $\mathrm{x}$ shows range of values whereas y represents soil moisture types. Figure 3 (a) Depicts the plot for low water management system (b) depicts the plot for medium water management system (c) depicts the plot for high water management system (d) depicts the plot for no water management system.

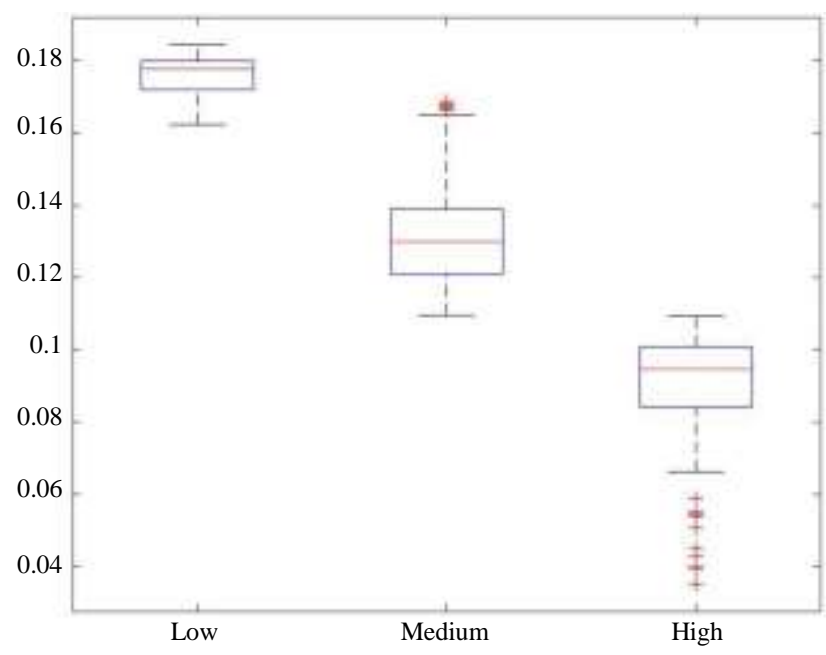

(a)

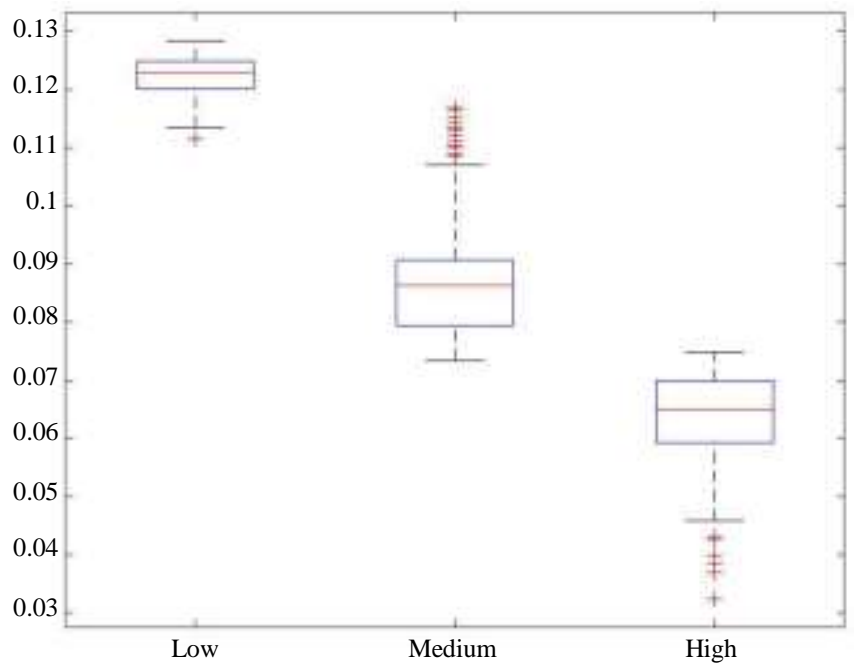

(b) 
Santhi Balachandran et al. / Journal of Computer Science 2020, 16 (4): 576.582 DOI: $10.3844 /$ jessp.2020.576.582

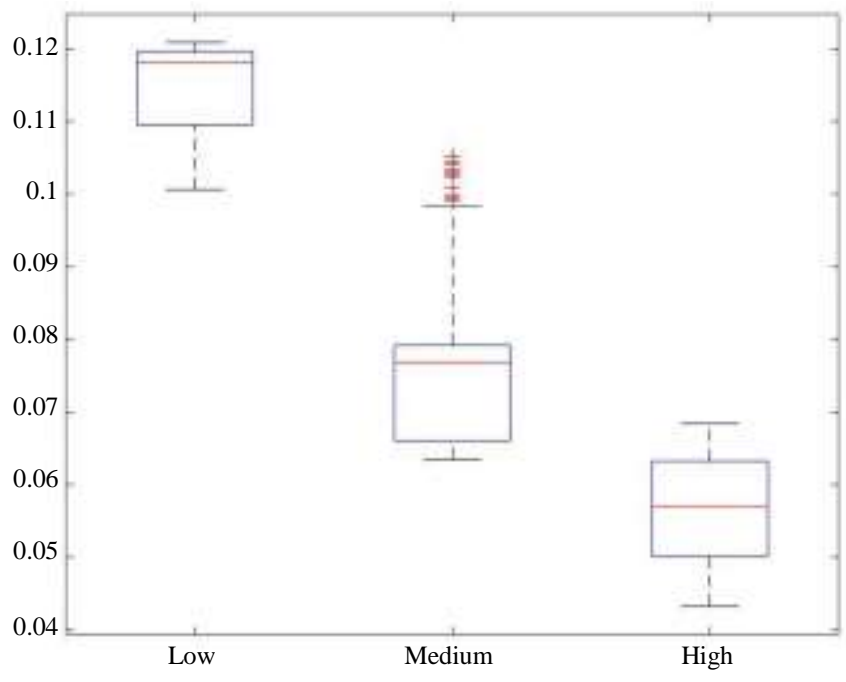

(c)

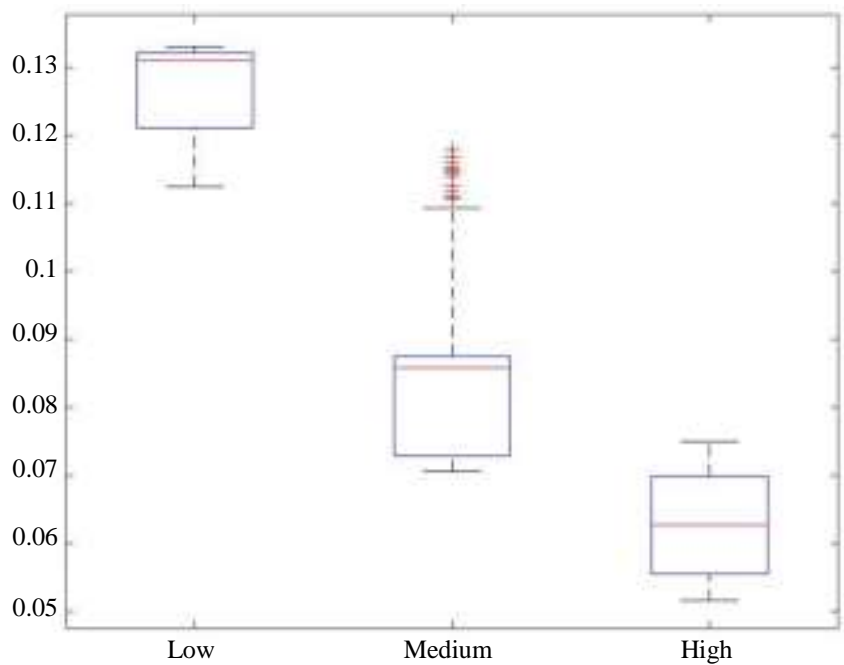

(d)

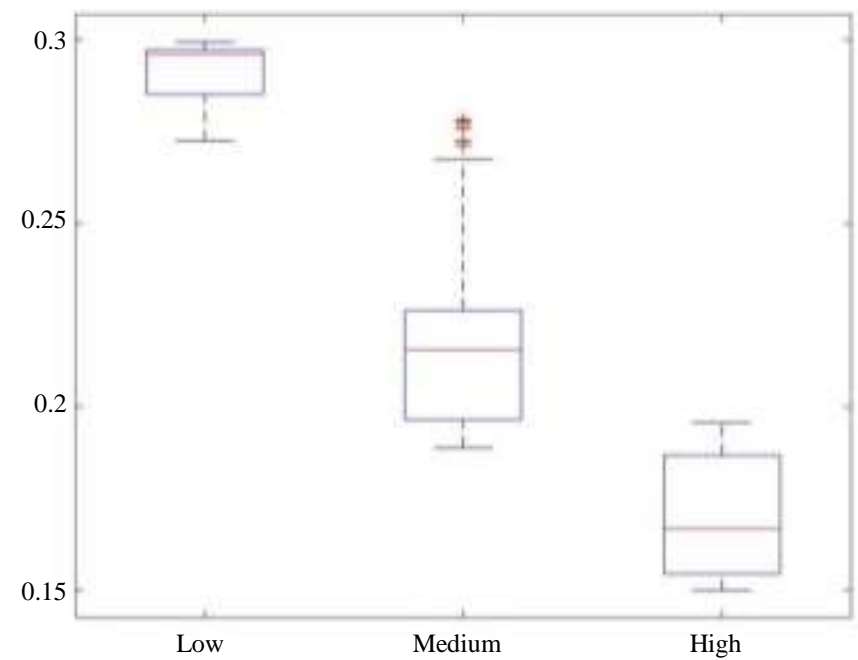

(e)

Fig. 2: Box plots of 454, 458, 494, 510 and 874 spectral band for soil moisture classification of hyperspectral data 

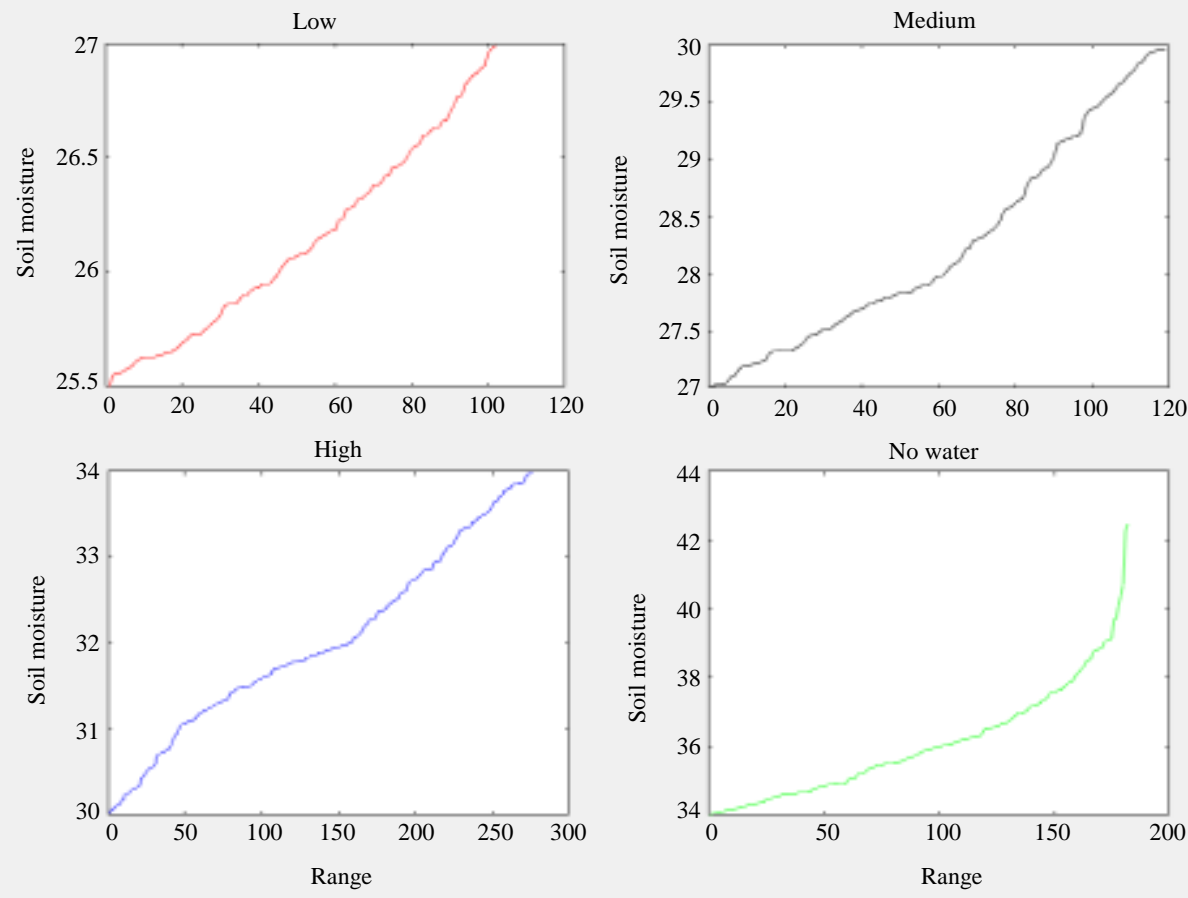

Fig. 3: Plot of range values

Table 5: Comparison result of classifications

\begin{tabular}{lll}
\hline METHOD & RMSE & $\mathrm{R}^{2}$ \\
\hline Decision Tree & 0.88861 & 0.94 \\
SVM & 0.55662 & 0.98 \\
LR & 1.46790 & 0.84 \\
\hline
\end{tabular}

Table 6: Soil moisture range value

\begin{tabular}{ll}
\hline Moisture Range & Linguistic variable \\
\hline $25 \leq x<26$ & LOW \\
$26 \leq x<29$ & MEDIUM \\
$29 \leq x \leq 33$ & HIGH \\
\hline
\end{tabular}

\section{Conclusion}

The contribution of this paper is to minimize the water wastage and improve the accuracy of water management. Data are collected from the experienced farmers as well as the researchers. Rules are framed from the collected data. These rules were used to make decisions in the fuzzy system. By taking the factors as input to the fuzzy system is Soil moisture. This input is given to the fuzzy inference system which chooses the amount of water for an optimum output Result from the different regression methods clearly states that SVM gives the better accuracy value. In Future, this system can be extended for the optimization of chemical wastage in farming using the fuzzy inference system.

\section{Acknowledgment}

We thank SASTRA Deemed University for providing the research facilities and infrastructure.

\section{Author's Contributions}

All authors are equally contributed in this work and this paper.

Santhi Balachandran: Planned and carried out the experiments.

Sourna Lakshmi: Participated in data collection and feature selection.

Nithya Rajendran: Participated in performance evaluation.

\section{Ethics}

This article is original and contains unpublished material. The corresponding authors have read and approve the manuscript and no ethical issues involved.

\section{References}

Bandos, T.V., L. Bruzzone and G. Camps-Valls, 2009. Classification of hyperspectral images with regularized linear discriminant analysis. IEEE Trans. Geosci. Remote Sens., 47: 862-873.

DOI: $10.1109 /$ TGRS.2008.2005729

Breiman, L., 2017. Classification and Regression Trees. Routledge. Giusti, E. and S. Marsili-Libelli, 2015. A fuzzy decision support system for irrigation and water conservation in agriculture. Environ. Modell. Software, 63: 73-86.

DOI: $10.1016 /$ j.envsoft.2014.09.020 
Cao, J., B. Zeng, J. Liu, Z. Zhao and Y. Su, 2017. A novel relocation method for simultaneous localization and mapping based on deep learning algorithm. Comput. Electrical Eng., 63: 79-90. DOI: 10.1016/j.compeleceng.2017.03.015

Cao, X., C. Wei, J. Han and L. Jiao, 2017. Hyperspectral band selection using improved classification map. IEEE Geosci. Remote Sens. Lett., 14: 2147-2151. DOI: 10.1109/LGRS.2017.2755541

Govender, M., K. Chetty and H. Bulcock, 2007. A review of hyperspectral remote sensing and its application in vegetation and water resource studies. Water Sa. DOI; 10.4314/wsa.v33i2.49049

Jadhav, B.D. and P.M. Patil, 2014. Hyperspectral remote sensing for agricultural management: A survey. Int. J. Comput. Applic.,

Khan, M.J., H.S. Khan, A. Yousaf, K. Khurshid and A. Abbas, 2018. Modern trends in hyperspectral image analysis: A review. IEEE Access, 6: 14118-14129. DOI: 10.1109/ACCESS.2018.2812999

Majone, B., F. Viani, E. Filippi, A. Bellin and A. Massa et al., 2013. Wireless sensor network deployment for monitoring soil moisture dynamics at the field scale. Proc. Environ. Sci., 19: 426-435.

DOI: 10.1016/j.proenv.2013.06.049

Martinez-Agirre, A., J. Álvarez-Mozos, H. Lievens and N.E. Verhoest, 2017. Influence of surface roughness measurement scale on radar backscattering in different agricultural soils. IEEE Trans. Geosci. Remote Sens., 55: 5925-5936.

DOI: 10.1109/TGRS.2017.2717043

Neamatollahi, E., J. Vafabakhshi, M.R. Jahansuz and F. Sharifzadeh, 2017. Agricultural optimal cropping pattern determination based on fuzzy system. Fuzzy Inform. Eng., 9: 479-491.

DOI: 10.1016/j.fiae.2017.12.004

Palacios-Orueta, A. and S.L. Ustin, 1998. Remote sensing of soil properties in the Santa Monica Mountains I. Spectral analysis. Remote Sens. Environ., 65: 170-183.

DOI: $10.1016 / \mathrm{S} 0034-4257(98) 00024-8$
Polak, A., T. Kelman, P. Murray, S. Marshall and D.J. Stothard et al., 2017. Hyperspectral imaging combined with data classification techniques as an aid for artwork authentication. J. Cultural Heritage, 26: 1-11. DOI: $10.1016 /$ j.culher.2017.01.013

Riese, F.M. and S. Keller, 2018. Hyperspectral benchmark dataset on soil moisture. Proceedings of the IEEE International Geoscience and Remote Sensing Symposium, Jul. 22-27, Valencia, Spain. DOI: $10.5281 /$ zenodo. 1227837

Roseline, P., C.J. Tauro and N. Ganesan, 2012. Design and development of fuzzy expert system for integrated disease management in finger millets. Int. J. Comput. Applic., 56: 31-36. DOI: 10.5120/8857-2815

Wark, T., P. Corke, P. Sikka, L. Klingbeil and Y. Guo et al., 2007. Transforming agriculture through pervasive wireless sensor networks. IEEE Pervasive Comput., 6: 50-57. DOI: 10.1109/MPRV.2007.47

Webber, H., T. Gaiser, R. Oomen, E. Teixeira and G. Zhao et al., 2016. Uncertainty in future irrigation water demand and risk of crop failure for maize in Europe. Environ. Res. Lett., 11: 074007-074007. DOI: $10.1088 / 1748-9326 / 11 / 7 / 074007$

Zhong, Y., L. Zhang, B. Huang and P. Li, 2015. An unsupervised artificial immune classifier for multi/hyperspectral remote sensing imagery. IEEE Trans. Geosci. Remote Sens., 44: 420-431. Hyperspectral imaging technique for plant leaf identification. Proceedings of the International Conference on Emerging Research in Electronics, Computer Science and Technology.

Zhong, Y., L. Zhang, B. Huang and P. Li, 2006. An unsupervised artificial immune classifier for multi/hyperspectral remote sensing imagery. IEEE Trans. Geosci. Remote Sens., 44: 420-431. DOI: $10.1109 /$ TGRS.2005.861548 